\title{
Les entérobactéries sécrétrices de béta-Lactamases à spectre étendu en urologie à
}

\author{
l'Hôpital Ibn Sina de Rabat.
}

\section{Extended spectrum beta-lactamase producing enterobacteriaceae in urology at Hospital}

\section{Ibn Sina of Rabat.}

Koné J, Bellahcen B, Awab A, El Moussaoui R, Alilou M, El Hijri A, Azzouzi A,

Service de Réanimation Chirurgicale, Hôpital Avicenne, CHU Ibn Sina, Rabat, Maroc

Faculté de Médecine et de Pharmacie de Rabat

Université Souissi Med V Rabat Maroc.

Auteur correspondant : Dr Joseph Koné, Service de Réanimation Chirurgicale, Hôpital Avicenne, CHU Ibn Sina, Rabat, Maroc Adresse email :drjosephkone@yahoo.fr

\begin{abstract}
Résumé :
L'antibiorésistance croissante des bactéries responsables des infections urinaires limite le choix des antibiotiques en chirurgie urologique. Parmi ces germes sont retrouvées les entérobactéries productrices de bétalactamases à spectre élargi (EBLSE) dont la progression devient inquiétante. L'objectif de cette étude était de déterminer la prévalence des entérobactéries sécrétrices de BLSE dans les prélèvements d'urines en péri opératoire de chirurgie urologique. Il s'agissait d'une étude rétrospective réalisée au centre hospitalier universitaire Ibn Sina de Rabat sur une période de 19 mois. N'ont pas été inclus dans cette étude, les prélèvements urinaires réalisés à dans un laboratoire externe. Les données étaient traitées avec le logiciel SPSS, les comparaisons faites par test du Khi deux ou de Student avec un seuil de risque alpha fixé à 5\%. Sur 830 prélèvements positifs 656 étaient des entérobactéries soit $79 \%$ des germes isolés. E.coli représentait 53,2\%, K.pneumoniae 28\%, E.cloacae 7,8\% et P.mirabillis 7\%. Les producteurs de BLSE représentaient 17,5\% de l'échantillon avec: E.coli $(12,3 \%)$, K.pneumoniae $(23,6 \%)$, E.cloacae $(39,2 \%)$ et P.mirabillis $(6,7 \%)$. On note une augmentation de la prévalence des entérobactéries BLSE entre 2008 et 2009 : respectivement de 14,4\% à 22,3\% des entérobactéries isolées; pour E.coli de $32,76 \%$ à $40,68 \%$ et pour K.pneumoniae de $34,48 \%$ à $42,37 \%$. Plusieurs facteurs de risque d'acquisition des EBLSE sont rapportés dans la littérature, dont principalement l'utilisation antérieure d'antibiotiques par ces patients. Une politique de gestion rationnelle des antibiotiques en ambulatoire et au sein des établissements de santé contribuera à une réduction de l'émergence des germes résistants.
\end{abstract}

Mots clés : EBLSE, Incidence, Infection, antibiorésistance, chirurgie urologique.

\section{Summary :}

Antibiotic resistance growth of bacteria responsible for urinary tract infections limits the choice of antibiotics in urological surgery. These germs are found producing enterobacteria extended spectrum beta-lactamases (ESBL) whose progress is worrying. The objective of this study was to determine the prevalence of ESBL-secreting enterobacteria in samples of urine in perioperative of urologic surgery. This was a retrospective study conducted at the University Hospital Ibn Sina in Rabat over a period of 19 months. Were not included in this study, urinary samples made in an external laboratory. The data were processed using SPSS software, the comparisons made by Chi-square test or two Student with an alpha risk threshold set at 5\%. 830 positive samples were 656 enterobacteria $79 \%$ of isolated germs. E.coli represented $53.2 \%$, 28\% K. pneumoniae, E.cloacae P.mirabillis $7.8 \%$ and $7 \%$. ESBL producers accounted for $17.5 \%$ of the sample with: E. coli $(12.3 \%)$, K. pneumoniae (23.6\%), E.cloacae (39.2\%) and P.mirabillis (6.7\%). There is an increase in the prevalence of ESBL enterobacteria between 2008 and 2009, respectively, from $14.4 \%$ to $22.3 \%$ of enterobacteria isolated; E.coli $32.76 \%$ to $40.68 \%$ and $34.48 \%$ for K. pneumoniae of $42.37 \%$. Several risk factors for ESBL acquisition are reported in the literature, mainly the previous use of antibiotics in these patients. A rational management policy for antibiotics in ambulatory care and in health facilities help to reduce the emergence of resistant organisms. Keywords: ESBLE, incidence, infection, antibiotic resistance, urological surgery. 


\section{Introduction :}

La présence d'une infection urinaire en préopératoire constitue une situation fréquente en chirurgie urologique. De plus en plus d'entérobactéries productrices de bétalactamases à spectre élargi (EBLSE) sont observées chez ces patients. Les EBLSE sont des entérobactéries productrices de bétalactamases capable d'hydrolyser les céphalosporines de $3^{\text {ème }}$ génération (C3G) [1]. Elles sont responsables d'infections potentiellement sévères; associées à une durée de séjour hospitalier plus longue et une augmentation de coûts. [2, 3,4] Elles constituent un véritable problème de santé publique à travers le monde [2]. On note une répartition inhomogène avec une prévalence inégale entre l'Amérique du Sud, l'Asie, l'Europe et les Etats-Unis [5]. Au Maroc, quelques études ont révélé des données sur les EBLSE uropathogènes. [6]. Une étude réalisée à Oujda au Maroc retrouvait une incidence de $16,7 \%$ d'entérobactéries BLSE chez des insuffisants rénaux [7]. De façon générale, l'antibiorésistance croissante limite le choix des antibiotiques [8] et justifie une surveillance épidémiologique. En péri opératoire, l'infection urinaire expose à des complications redoutables [9], allant des infections du site opératoire à des états septiques graves, impliquant de ce fait une augmentation de la morbidité et un surcout de soins. La question reste un sujet majeur d'actualité vu les défis thérapeutiques pour la prise en charge des EBLSE. Les objectifs de notre étude étaient de connaitre la prévalence des EBLSE et d'identifier les germes en cause en préopératoire de chirurgie urologique.

Malades et méthodes :

Il s'agissait d'une étude rétrospective sur période de 19 mois allant de Janvier 2008 à Juillet 2009. Elle a porté sur les prélèvements urinaires réalisés chez les patients hospitalisés en attente d'une intervention chirurgicale aux services d'urologie A et B du centre hospitalier universitaire Ibn Sina de Rabat. Les examens bactériologiques urinaires ont été effectués au laboratoire du même centre. Les prélèvements d'urine étaient réalisés avec des tubes stériles fournis par le laboratoire, après désinfection de la région périnéale ou du tube collecteur (pour les patients sondés). Les échantillons ont été immédiatement acheminés au laboratoire. L'examen cytobactériologique recherchait à l'examen direct une leucocyturie et d'autres éléments figurés (cellules, hématies, cristaux...), et d'une bactériurie (uroculture et dénombrement des germes). La bactériurie a été définie par l'identification de germes avec un nombre supérieur ou égal à $10^{5} \mathrm{UCF} / \mathrm{ml}$ d'urines avec au plus deux espèces différentes, sans tenir compte du nombre de leucocytes urinaires. Les galeries API $20^{\mathrm{E}}$ ont été utilisées pour l'identification des germes. Les tests de sensibilité étaient réalisés selon les procédures du Comité de l'antibiogramme de la Société française de microbiologie (CASFM). Les souches de BLSE étaient détectées par le test de synergie entre un disque central d'amoxicilline + acide clavulanique à une distance de $30 \mathrm{~mm}$ de ceux de céfepime, céfotaxime, ceftazidime, et d'aztréonam. La présence de BLSE était retenue devant l'aspect caractéristique en "bouchon de champagne ». Les principales variables étudiées étaient: la positivité des examens urinaires, les germes isolés, la production de BLSE, la date du prélèvement. La prise préalable d'antibiotique, les motifs d'hospitalisation et la présence d'un matériel urinaire (sonde urinaire à demeure, sonde $\mathrm{J}$ et autres) n'ont pas été pris en compte. Les données ont été saisies et analysées avec le logiciel SPSS 20.0 (IBM Statistics. Inc), et exprimées en effectifs et pourcentages. Les comparaisons ont été faites avec le test de Khi-2 avec un seuil de significativité à $5 \%$. Les résultats sont présentés sous forme de tableaux et graphiques, puis discutés avec les données de la littérature.

\section{Résultats}

Nous avons colligés 830 prélèvements positifs examinés au laboratoire, dont 656 étaient des entérobactéries soit 79,03\% (Tableau I). Escherichia coli était le plus fréquent avec 53,2\%, suivi de Klebsiella pneumoniae ( 28\%), de Enterobacter cloacae (27,59\%), de Protéus mirabillis, de Citrobacter freundii avec $1.7 \%$ chacun. Les autres entérobactéries étaient Morganella, Serratia, Providencia et Salmonella avec respectivement $6,5,2$ et 1 isolats. Les entérobactéries productrices de BLSE représentaient $17,5 \%$ de l'échantillon. Le profil des entérobactéries est résumé sur le tableau II. Les entérobactéries BLSE sont répertoriées selon la période de l'année sur le tableau III et le graphique 1. La répartition sur les deux années montrait que sur 349 isolats d'E.Coli 43 étaient producteurs de BLSE ; cette fréquence était de 45 isolats BLSE sur 183 pour K. pneumoniae ; 20 isolats BLSE sur 51 pour Enterobacter; 3 isolats BLSE sur 45 pour Proteus et 4 sécréteurs de BLSE sur 15 isolats de Citrobacter. Les souches de Serratia, Morganella, Salmonella et Providencia isolées dans notre échantillon n'étaient pas productrices de BLSE.

\section{Discussion}

Depuis la découverte de la première bétalactamase dans les années 1940, d'autres types d'enzymes capables d'hydrolyser les pénicillines et certaines céphalosporines ont été décrites jusqu'à la commercialisation des C3G en 1980 [2]. En 1988, Knothe et al retrouvaient dans une étude une résistance de certaines souches de K. pneumoniae et Serratia marcescens à la cefotaxime, cefoxitine, céfamandole et cefuroxime [10]. Il s'agissait d'un 
pouvoir de production d'autres nouvelles bétalactamases, dotées du pouvoir d'hydrolyser les C3G d'où l'appellation de «bétalactamases à spectre élargie » [1]. Néanmoins, celles-ci ne conféraient pas aux entérobactéries une résistance aux céphamycines et aux carbapénèmes. [4, 11-12]. Une production de carbapanémases a été récemment décrite, due à l'utilisation abusive des carbapénèmes, d'où les recommandations dans ce sens [13-14]. De nouvelles alternatives thérapeutiques sont nécessaires, comme la céfoxitine (céphamycine), la témocilline, et avibactam. [15-16]. Les EBLSE sont responsables d'infections sévères prédominant sur les sites urinaires, respiratoires. [4, 17]. L'appareil urinaire était le site le plus fréquemment touché. Dans une étude portant sur les infections nosocomiales Razine R. et al ont trouvé l'incidence de l'infection urinaire à 35\%. [17]. L'augmentation de l'incidence des EBLSE pose un véritable problème de santé publique dans plusieurs régions du monde. Il existe plus de 90 types de bêtalactamases TEM (pour TEMoniera le nom de la première patiente chez qui cette souche a été isolée) et plus de 25 types de bêtalactamases SHV (sulfhydryl variable : il s'agit des souches chez lesquelles les réactifs sulfhydryles ont un effet variable sur la spécificité du substrat) [18]. Dans cette étude, nous n'avons pas évalué les paramètres comme l'origine nosocomiale ou communautaire, les motifs d'hospitalisations ou les pathologies urinaires associés, les suites périopératoires des patients. L'analyse des prélèvements urinaires ne tenait pas compte de la présence ou non d'un matériel invasif comme une sonde à demeure, un cathéter urétéral, ou de sondes en J. Il s'agissait surtout de connaitre la prévalence et de suivre l'évolution de celle-ci sur deux années successives. Nous avons trouvé que sur 860 prélèvements positifs au laboratoire, $656(79,03 \%)$ étaient des entérobactéries dont $115 \quad(17,5 \%)$ productrices de BLSE. Les EBLSE ont fait l'objet de plusieurs études évaluant la prévalence et les facteurs de risque. [2, 19]. Le profil de résistance est une source d'inquiétude quant à l'utilisation rationnelle des antibiotiques [20]. On note une augmentation de l'incidence des EBLSE entre 2008 et 2009. Ce constat est fait par d'autres auteurs [21,22]. Dans une étude de Fouquet et al avec trouvaient une répartition des EBLSE avec majoritairement E. coli $(66,7 \%), K$. pneumoniae $(11,1 \%)$ et $E$. cloacae $(11,1 \%)$, suivi d'un P.mirabilis, un Klebsiella oxytoca, un $S$. marcescens avec $03 \%$ chacun. [21]. En général, nous avons trouvé une différence statistiquement significative entre l'incidence des entérobactéries (14,4\% en 2008 versus $22,3 \%$ en 2009 ; soit une incidence globale de $17,5 \%$. Les souches de Serratia marcescens, Morganella morganii,
Salmonella et Providencia stuartii isolées n'étaient pas productrices de BLSE. Dans une méta-analyse de 26 études réalisées dans 13 pays en Afrique, Tansarli et al. ont trouvé des souches productrices de BLSE avec de faibles prévalences [22]. En tenant compte des données de la littérature et de notre étude, il y a une tendance à l'augmentation rapide de l'incidence des EBLSE urinaires. El Bouamri MC et all, ont trouvé que cette augmentation devenait inquiétante, vu le profil de résistance retrouvé dans ces études [8] Avec l'émergence de germes producteurs de carbapénemases, la commission des anti-infectieux de l'Assistance Publique-Hôpitaux de Paris, a émis des recommandations concernant l'utilisation des carbapénèmes qui doit obéir à quatre règles d'usage : bacilles à Gram négatif multirésistants en milieu hospitalier ; absence d'alternative ; réévaluation dans les 48 heures si traitement empirique pour une désescalade thérapeutique ; l'ertapénème pour les infections urinaires à entérobactéries productrices de bêtalactamases à spectre élargi [23]. Les recommandations suggèrent la nécessité de mise en place d'une politique de prévention dans les unités de soins, une stratégie d'antibiothérapie et de prévention de l'émergence des résistances bactériennes [24-26].

\section{Conclusion :}

La présence des EBLSE pose un véritable problème en péri-opératoire de chirurgie urologique. La prévalence était de $17,5 \%$, avec une augmentation significative entre 2008 et 2009. Klebsiella pneumoniae et Escherichia coli étaient les souches prédominantes. Le profil de résistance de ces entérobactéries limite considérablement le choix des antibiotiques. Des études ultérieures sont nécessaires pour explorer l'importance des EBLSE en fonction des différentes pathologies urinaires, de la présence ou non d'un matériel invasif urinaire. Le bon usage des antibiotiques et le respect des recommandations permettraient une réduction significative de l'incidence des germes multi résistants.

\section{Bibliographie}

1. Philippon A, Labia R, Jacoby G. Extendedspectrum béta-lactamases. Antimicrob Agents Chemother 1989; 33(8):1131-6.

2. Vodovara D, Marcadé G, Raskine L, Malissin I, Megarban B. Entérobactéries productrices de bétalactamases à spectre élargi : épidémiologie, facteurs de risque et mesures de prévention. La Revue de médecine interne 2013 ; 34(11): 687-693

3. Yang $\mathrm{YS}, \mathrm{Ku} \mathrm{CH}$, Lin $\mathrm{JC}$, et al. Impact of extended spectrum $\quad \beta$-lactamase-producing Escherichia coli and Klebsiella pneumoniae on the outcome of community-onset bacteremic urinary 
tract infections. J Microbiol Immunol Infect 2010; 43(3):194-9.

4. Forestier E. Actualités sur les infections urinaires et respiratoires basses du sujet âgé. Cah. Année Gérontol 2014; 6 (3):119-125

5. Reinert RR, Low DE, Rossi F, Zhang X, Wattal C, Dowzicky MJ. Antimicrobial susceptibility among organisms from the Asia/Pacific Rim. Europe and Latin and North America collected as part of TEST and the in vitro activity of tigecycline. J Antimicrob Chemother 2007; 60(5):1018-29.

6. Azouzzi A. Résistance aux antibiotiques: Qu'en pensent les spécialistes? http:// pharmacie.ma/uploads/ pdfs/ pr-abderrahimazzouzi -infosante_mars_2014.pdf

7. Chemlal A, Ismaili FA, Karimi I et al. Les infections urinaires chez les patients insuffisants rénaux chroniques hospitalisés au service de néphrologie: profil bactériologique et facteurs de risque. The Pan African Medical Journal. 2015;20:100.

8. El Bouamri MC, Arsalane L, Kamouni

$\underline{Y}, \underline{\text { Berraha M}}$, Zouhair S. Evolution récente du profil épidémiologique des entérobactéries uropathogènes productrices de bêta-lactamases à spectre élargi à Marrakech, Maroc. Progrès en urologie. 2014; 24(7) : 451-455

9. Lahlou A, Chegri M, L'Kassmi H. Epidémiologie et résistance aux antibiotiques des entérobactéries isolées d'infections urinaires à l'hôpital militaire Moulay-Ismail de Meknès. Antibiotiques 2009; 11(2):90-96.

10. Knothe H, Shah P, Krcmery V, Antal M, Mitsuhashi S. Transferable resistance to cefotaxime, cefoxitin, cefamandole and cefuroxime in clinical isolates of Klebsiella pneumoniae and Serratia marcescens. Infection1983; 11 (6):315317.

11. Prakash V, Lewis JS, Herrera ML, Wickes BL, Jorgensen JH. Oral and parenteral therapeutic options for outpatient urinary infections caused by enterobacteriaceae producing CTX-M extendedspectrum beta-lactamases. Antimicrob Agents Chemother 2009; 53(3):1278-1280.

12. Nicolau DP. Carbapenems: a potent class of antibiotics. Expert Opin Pharmacother. 2008; 9(1): 23-37

13. Sibhghatulla $S$, Jamale F, Shazi $S$, Syed $M$, Danish R, Amjad KM. Antibiotic resistance and extended spectrum beta-lactamases: Types, epidemiology and treatment. Saudi J Biol Sci. 2015; 22 (1): 90-101.

14. Nordmann P. Carbapenemase-producing Enterobacteriaceae: overview of a major public health challenge. Med Mal Infect. 2014; 44(2): 516

15. Clouzeau B, Boyer A, M'Zali F, Kann M, Gruson D. Nouvelles stratégies thérapeutiques contre les entérobactéries productrices de $\beta$ lactamases à spectre étendu. Réanimation 2015; 24(3) :270-277.

16. Asakura $\mathrm{T}$, Ikeda $\mathrm{M}$, Nakamura $\mathrm{A}$, et al. Efficacy of empirical therapy with noncarbapenems for urinary tract infections with extended-spectrum $\quad \beta$-lactamase-producing Enterobacteriaceae. Int J Infect Dis 2014; 29: 91-5 17. Razine R, Azzouzi A, Barkat A et al. Prevalence of hospital-acquired infections in the university medical center of Rabat, Morocco. Int Arch Med. 2012; 5(1):26

18. Paterson DL, Bonomo RA. Extended-spectrum béta-lactamases: a clinical update. Clin Microbiol Rev 2005;18(4):657-86.

19. Sekhsokh Y, Chadli M, El Hamzaoui SA. Fréquence et sensibilité aux antibiotiques des bactéries isolées dans les urines. Médecine et maladies infectieuses 2008; 38(1): 324-327

20. Tlamçani Z, Ellaia K, Benomar A, Kabbaj H, Alaoui AE, Seffar M. La résistance aux fluoroquinolones chez des souches de Klebsiella spp productrices de bêtalactamase à spectre étendu isolées dans les urines. Ann Biol Clin 2009; 67(5) : 553-556

21. Fouquet M, Morange V, Bruyere F. Évolution sur 5 ans des infections à germes produisant une betalactamases à spectre étendu. Prog Urol 2012;22:17-21.

22. Tansarli GS, Poulikakos B, Kapaskelis A, Falagas ME. Proportion of extended-spectrum $\beta$ lactamase (ESBL)-producing isolates among Enterobacteriaceae in Africa: evaluation of the evidence-systematic review. J Antimicrob Chemother 2014; 69 (5):1177-84.

23. Gauzit R, Gutmann L, Brun-Buisson C. Recommandations de bon usage des carbapénèmes. Antibiotiques 2010; 12(4): 183-189

24. Lefort A, Nicolas CMH. Les entérobactéries productrices de $\beta$-lactamases à spectre étendu (BLSE) et les céphalosporines de troisième génération en 2012. Journal des Anti-infectieux 2012; 14(2):51-57

25. Brun-Buisson C. La maîtrise de la diffusion des bactéries multi-résistantes endémiques en réanimation: enseignements des récentes études. Journal des Anti-infectieux 2014; 16(3): 144-153 26. Stratégie d'antibiothérapie et prévention des résistances bactériennes en établissement de santé. Médecine thérapeutique. 2009;15(2):157-168. 
Tableau I : Répartition des bactéries isolées

\begin{tabular}{|c|c|c|c|c|}
\hline & 2008 & 2009 & Total & \multirow{3}{*}{$\begin{array}{l}\text { * : exprimés en } \\
\text { Effectifs } \\
\text { (pourcentages) }\end{array}$} \\
\hline 1 1уре bacteries & $n=506$ & $n=324$ & $\mathrm{n}=\mathbf{8 3 0}$ & \\
\hline Entérobactéries * & $396(78,26 \%)$ & $260(80,25 \%)$ & $656(79,03 \%)$ & \\
\hline Non Entérobactéries* & $110(21,74 \%)$ & $64(19,75 \%)$ & $174(20,96 \%)$ & \\
\hline
\end{tabular}

Tableau II : Répartition des Entérobactéries isolées selon le profil BLSE

\begin{tabular}{lllll}
\hline Période & Total & 2008 & 2009 & Valeur de $\mathbf{p}$ \\
& $(\mathrm{n}=656)$ & $(\mathrm{n}=396)$ & $(\mathrm{n}=260)$ & \\
\hline EBLSE & $\mathbf{1 1 5}$ & $\mathbf{5 7 ( 1 4 , 3 9 \% )}$ & $\mathbf{5 8}(\mathbf{2 2 , 3 \% )}$ & $\mathrm{P}<0,0001$ \\
Non EBLSE & 541 & $339(85,61 \%)$ & $202(77,79 \%)$ & $\mathrm{P}<0,001$
\end{tabular}

Graphique I : Répartition des EBLSE isolées selon l'espèce ( $\mathrm{n}=115)$ sur les deux années

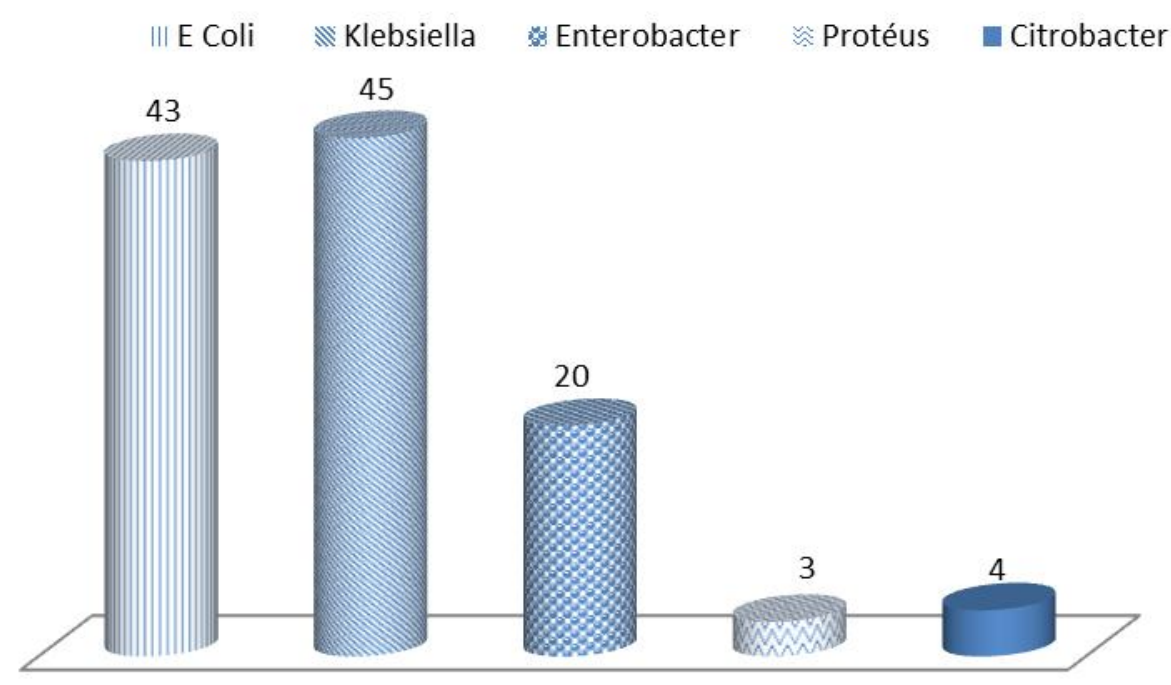


Tableau III : Répartition des BLSE selon la période.

\begin{tabular}{lrrrrr}
\hline \multirow{2}{*}{ Germes } & \multicolumn{2}{c}{$\mathbf{2 0 0 8}(\mathbf{n = 5 8})$} & \multicolumn{2}{c}{$\mathbf{2 0 0 9}(\mathbf{n = 5 9})$} & \multirow{2}{*}{ Valeur de $\boldsymbol{p}$} \\
\cline { 2 - 5 } & EBLSE & $\boldsymbol{\%}$ & EBLSE & $\boldsymbol{\%}$ & \\
\hline E.coli & 19 & 32,76 & 24 & 40,68 & 0,142 \\
K.pneumoniae & 20 & 34,48 & 25 & 42,37 & 0,047 \\
E.cloacae & 16 & 27,59 & 4 & 6,78 & 0,009 \\
P.mirabillis & 1 & 1,72 & 2 & 3,39 & 0,472 \\
C. freundii & 1 & 1,72 & 3 & 5,08 & 0,472
\end{tabular}

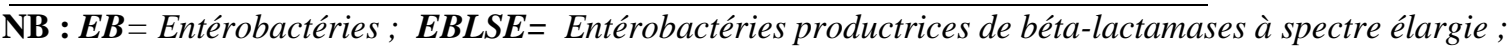

$\%=$ pourcentage 